\title{
Anisotropic etching in (311) Si to fabricate sharp resorbable polymer microneedles carrying neural electrode arrays
}

\author{
Frederik Ceyssens ${ }^{1^{*}}$, Marleen Welkenhuysen ${ }^{3}$, Robert Puers ${ }^{1}$ \\ ${ }^{1}$ KULeuven dept. ESAT-MICAS. Kasteelpark Arenberg 10, Leuven, Belgium. \\ 2 Imec vzw, Kapeldreef 75, Leuven, Belgium. \\ *Corresponding author: fceyssen@esat.kuleuven.be \\ Other authors' email adresses: Marleen.Welkenhuysen@imec.be, \\ robert.puers@esat.kuleuven.be
}

\begin{abstract}
Polymer microneedles that get resorbed after insertion in the body have several interesting applications, for example in the insertion of ultra-flexible electrode arrays in neural tissue. In this work, we explore the use of molds created by etching in (311) silicon as a basis for the fabrication of such microneedles. Such molds can be etched anisotropically to much sharper angles compared to standard (100) silicon. It is demonstrated that sharp PLGA microneedles can be fabricated using the molds. It is also shown that by simple thermal bonding the fabricated PLGA microneedles can be combined with a ultra-flexible polyimide-based thin-film electrode array.
\end{abstract}

\section{Introduction}

Communicating directly with the nervous system by applying and measuring electric pulses is a topic that has inspired scientists ever since the discovery of bioelectricity by Alessandro Galvani in 1791. As progress grew, miniaturized electronics and fine wire electrodes became commonplace in experimental neuroscience. A major step in increasing the resolution of the recordings was the advent of micromachined electrode arrays, often in needle shape, to allow insertion in the brain to areas close to the neuron cell bodies [1]. Currently, neural electrode arrays with integrated electronics are becoming commercially available, but are not widely employed yet in neuroscience.

When the goal of the research is long term (chronic) interfacing, such as for example to steer an advanced prosthetic device, the in vivo lifetime of the implant becomes an important issue. The lifetime is determined by factors such as the degradation of the insulation resistance and the evolution of the electrode impedance [2]. Another factor is the encapsulation of the implant by scar tissue, which increases the distance to the nearest viable neurons and thus limits the available resolution in recording while also hampering stimulation. Minimisation of scar tissue, allowing neural recording implants that last a lifetime, is the main long-term goal of the work presented here. 
The formation of the so-called glial scar around a foreign object in the brain is relatively well documented [3][4][5]. Damaged cells and pro-inflammatory factors from damaged blood vessels attract immunoactive cells such as microglia that try to remove the source of the inflammation. The release of pro-inflammatory factors attracts more immune cells and opens the blood-brain barrier, a process of positive feedback. If the source of the inflammation persists nevertheless, astrocyte cells start to form a dense layer around the object, shielding it from the brain. Turner et al. [6] have shown that this process leaves a permanent cavity if the object is removed after 4 weeks or more.

At this point, substantial evidence has been gathered that reducing the mechanical damage exerted by the implant during insertion and during its operation is very important to reduce scar formation. To avoid chronic mechanical irritation caused by movement of the brain parenchyma relatively to the implant, several measures can be taken. Firmly anchoring the implant to the skull should be avoided [7]. Also, the implant should be made compliant enough to move along with the brain by reducing its cross section and using compliant materials [8]. Using ultra-compliant, submicrometer thick SU-8 based implants, Lieber et al. have shown recently that it is possible to interweave electrodes and neural tissue without the formation of any scar tissue [9].

However, these results were achieved by injecting these thin, mesh-shaped electrodes in the brain using a fine glass syringe. Another method would be to use a resorbable carrier, which could allow a more precise control of the electrode positions. Several resorbable materials have been reported in literature, including maltose [10], PEG [11], silk [11][12], chitosan[13] and extracellular matrix proteins [14]. In this work, we will investigate the use of short-chained PLGA (Poly Lactic-coGlycolic Acid), which is a well established material in medical applications such as drug delivery. However, no application related to the delivery of neural electrodes was reported yet.

Moreover, in the field of neural implants, the resorbable materials have been mainly applied using simple dip coating techniques. In other fields, such as microneedles for drug delivery, more advanced approaches based on e.g. microfabricated silicon molds have been investigated already [15][16][17][18][19][20].

In this work, we will use such molds as well, but furthermore improve their sharpness drastically by introducing (311) oriented silicon to the process instead of the common (100) variant. A micromachining process that allows combining lithographically fabricated thin film electrode arrays with needles micromolded in (311) silicon is presented, together with preliminary in vitro measurement results.

\section{Fabrication process}

\subsection{Nonresorbable backbone fabrication}

The nonresorbable backbone, containing the electrodes, metal connections and an upper and lower polyimide isolation layer is fabricated using a micromachining processes published earlier [22]. A design was used that features four $12 \mu \mathrm{m}$ diameter electrodes in a tetrode configuration, in line with two $32 \mu \mathrm{m}$ diameter electrodes that can serve as reference or as stimulation electrode (Figure 1, upper right). 
Four inch silicon wafers are cleaned and a $150 \mathrm{~nm}$ thick aluminium layer is deposited as a sacrificial layer (Figure 1, steps 1-2). Then, a polyimide (PI) layer is deposited by spin coating (PI2611, diluted with NMP in a weight ratio of $1: 1.6,3000 \mathrm{rpm}$ ), (step3). The layer is partially cured at $200{ }^{\circ} \mathrm{C}$ according to our protocol designed to improve insulation lifetime [22]. In a next step, a patterned platinum layer is deposited using lift-off processing and sputter coating (step 4). A second PI layer is then spun on and cured at $350{ }^{\circ} \mathrm{C}$ (step 5). After spin coating and patterning a positive photoresist, reactive ion etching ( $R I E)$ is then used to pattern the polyimide, defining the outline of the electrode array (step 6). As the RIE stops on the platinum as well as on the aluminium layer, the electrodes and bondpads can be opened in the same etch step. Bonding and release (steps 7 and 8 ) are discussed in paragraph 2.4 .

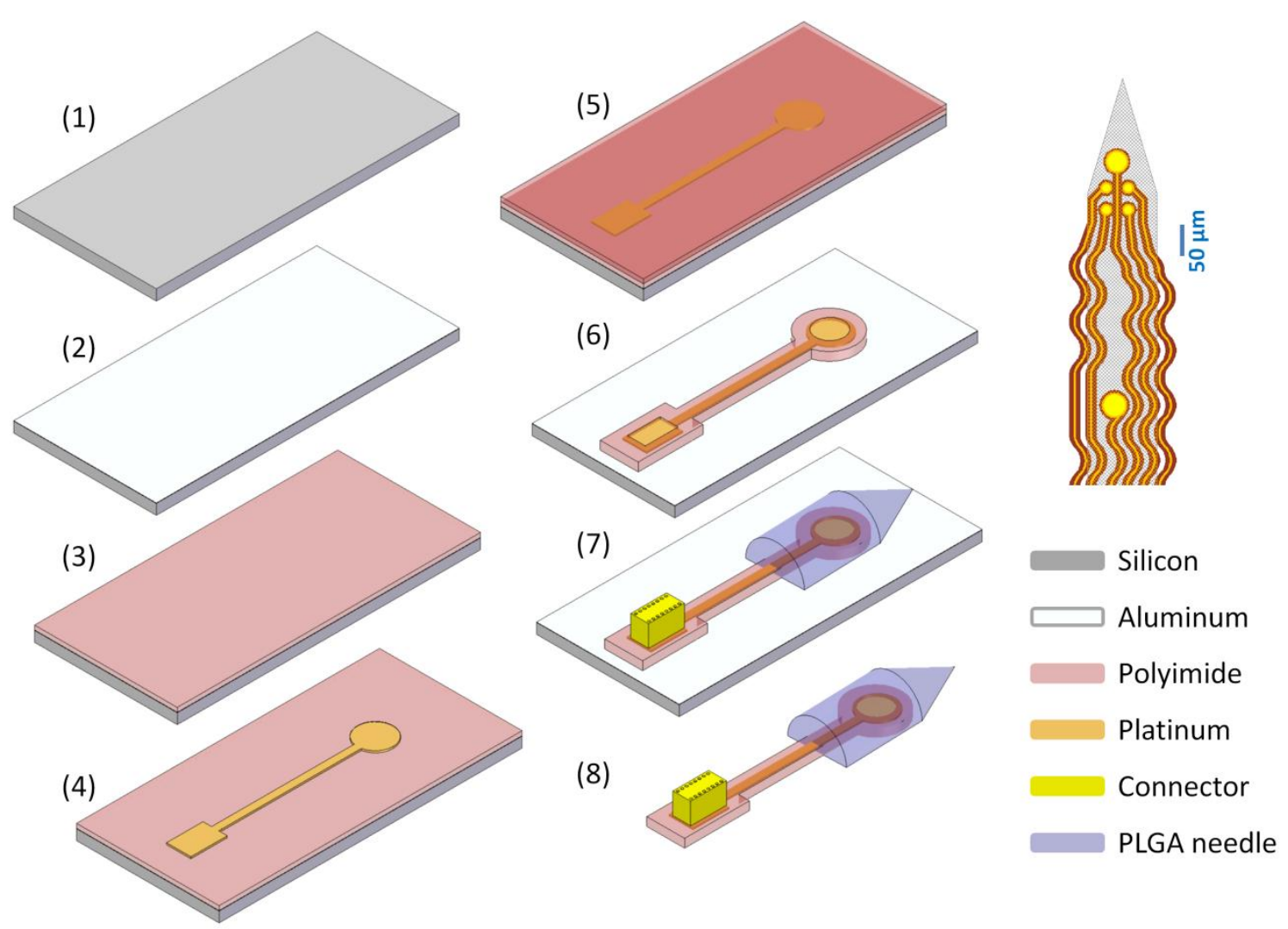

Figure 1: Steps 1-8: fabrication sequence of non-resorbable backbone and assembly of connector and PLGA microneedle. Top right: electrode layout. Note that needle will get larger than drawn due to underetching of the vertical lines defining the shaft.

\subsection{Etching in (311) silicon}

It is well known that it is possible to create pyramidal pits in (100) silicon by anisotropic wet etching, which reveals the non-etching (111) planes. However, as the angle between the (111) planes and the surface is $54.7^{\circ}$, if used to create molds for the fabrication of needles this yields rather blunt devices. We therefore decided to investigate anisotropic etching in (311) silicon for this purpose [23][24]. (311) silicon is rarely used, but is still available commercially (Sil'Tronix, France). 
The orientation of the intersections of the (111) planes and the (311) wafer surface was calculated to be at an angle of $\pm 16.78^{\circ}$ from the (110) wafer flat, as well as $\pm 16.78^{\circ}$ from the line perpendicular to the flat. This is displayed graphically on the top part of figure 2. It can be seen that it is possible to etch cavities with a sharp point oriented towards the right side of the wafer.

The calculated angles were verified in the etch experiment described below. In this experiment, molds were fabricated that were thereafter employed for the fabrication of sharp PLGA microneeldes. For this experiment, the mask layout shown on the bottom part of figure 2 was used.

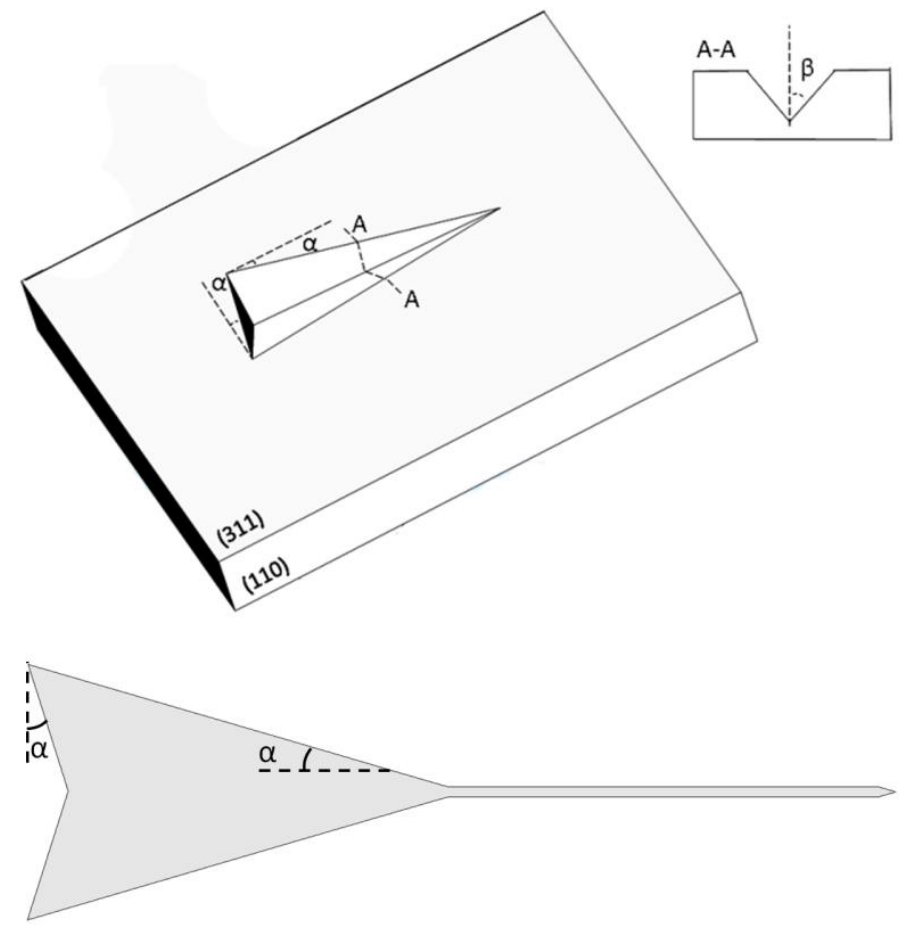

Figure 2, top: (311) wafer with three non-etching (111) planes revealed. $\alpha=16.78^{\circ} . \quad b=27.1^{\circ}$. A fourth non-etching plane exists, at an angle of - $\alpha$ from the line perpendicular to the (110) flat.

Bottom: Mask layout for (311) Si etch and mold experiment. Another version with a vertical edge to the left was also included. Note that the horizontal lines will be underetched, resulting in wider needles shafts than designed.

(311) Silicon wafers were cleaned in an ultrasonic bath in DI water, and then in piranha and in $1 \% \mathrm{HF}$. To etch the mold in (311) silicon, first a 2 micron thick wet thermal oxide was grown at $1200{ }^{\circ} \mathrm{C}$ for 6.5 hours.

The results of the anisotropic etch experiments is shown in figure 3. In the first etch experiments, etching was done in $30 \% \mathrm{KOH}$ at $60^{\circ} \mathrm{C}$. $\mathrm{KOH}$ was preferred over TMAH as it is has reduced toxicity [25], though nevertheless in both cases appropriate safety measures need to be taken to allow handling the chemicals safely. However, this comes at the price of reduced selectivity towards the oxide mask [26]. The non-etching planes were as expected on figure 2. Downward etching proceeded at $0.41 \mu \mathrm{m} / \mathrm{min}$. Horizontal lines (i.e. parallel with the flat) underetched at a rate of $0.21-0.24$ $\mu \mathrm{m} / \mathrm{min}$, but in a somewhat rough pattern as is clear from the underetch variation. The sidewalls thus are under a $58.8^{\circ}$ angle with the wafer surface. Vertical lines (i.e. perpendicular to the flat) created a profile with a $32.7^{\circ}$ angle with the wafer surface. The bottom surface was also quite rough, having a Ra of $100 \mathrm{~nm}$. 
In further experiments, a switch was made to $42.5 \% \mathrm{KOH}$ in order to reduce roughness. This reduced bottom surface roughness to a Ra of $60 \mathrm{~nm}$ (figure 3, middle). The downwards etching rate was measured to be $0.31 \mu \mathrm{m} / \mathrm{min}$ at that concentration.

After stripping the oxide mask, a thin silicon overhang was still visible at the upper edge of the etched pits. This would clearly hinder demolding, and is therefore removed with a short reactive ion etch step ( 3 minutes at $100 \mathrm{~W}, 90 \mathrm{sccm} \mathrm{SF}_{6}, 10 \mathrm{sccm} \mathrm{O}_{2}, 80 \mathrm{mTorr}$ pressure, 6 inch platen diameter).
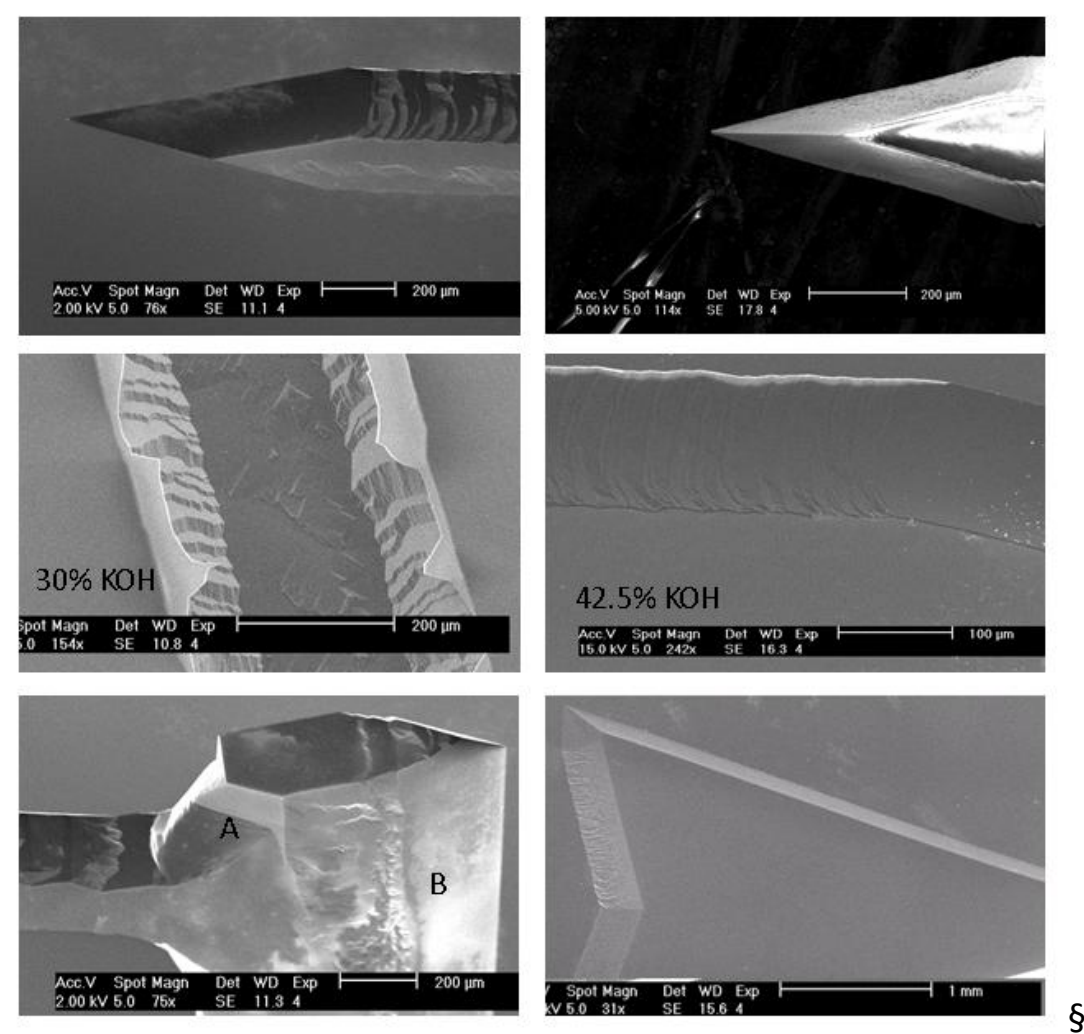

Figure 3: Results from etching in (311) silicon. Top left: sharp tipped trench formed out of intersecting (111) planes. Top right: PLGA needle molded by of the trench tip (see section 2.3). Middle: showing the decrease in shank roughness when switching to a higher $\mathrm{KOH}$ concentration. Bottom left: showing the undercut of convex right corners ( $A$ ) and the small angle plane showing near a vertical line (B). Bottom right: needle base defined only using (111) planes, to eliminate underetch and roughness. Note that in these pictures, the wafer flat is located horizontally, above the field of view.

\subsection{Molding of microneedles in (311) silicon}

Our molding process to fabricate sharp microneedles is summarized in figure 4 . Wafers etched in $42.5 \% \mathrm{KOH}$ as described in section 2.2 were used as molds. To form a sacrificial layer to release the supporting needles, a Ti / Al stack was deposited by sputter coating. (3' Ti sputtering at 300W, 35' Al sputtering at $200 \mathrm{~W}$ for a layer thickness of $2.2 \mu \mathrm{m}, 3^{\prime \prime}$ targets).

PLGA (Purasorb PDLG 5002A) was then melted on the wafer at $130^{\circ} \mathrm{C}$ and spread using a scraper while the wafer was cooling down on the hotplate. After filling the mold cavities, the assembly was 
heated up tot $130{ }^{\circ} \mathrm{C}$ once more and a fresh scraper was used to remove excess PLGA. Then, the wafer was mounted on a carrier wafer using wax, and polished from the front side over a series of rotating wet polishing mats, ranging from grid 80 to 1000 . This removed all PLGA from the surface, leaving only PLGA in the mold cavities.

In a final step, the needles were released from the mold by submerging in $7.5 \% \mathrm{HCl}$ overnight. An acidic release step was preferred as the decomposition of PLGA is a reaction that releases lactic acid, and is thus expected to be slowed down in a low pH environment as per Le Chatelier's principle. This was verified experimentally as well: PLGA beads were bathed in $\mathrm{HCl}$ for one week and seemed undamaged, while the same beads did dissolve in a $0.1 \mathrm{NaOH}$ solution.

It was possible to use an ultrasonic bath to remove the needles that did not dislodge spontaneously. The wafer could be cleaned with piranha for re-use.
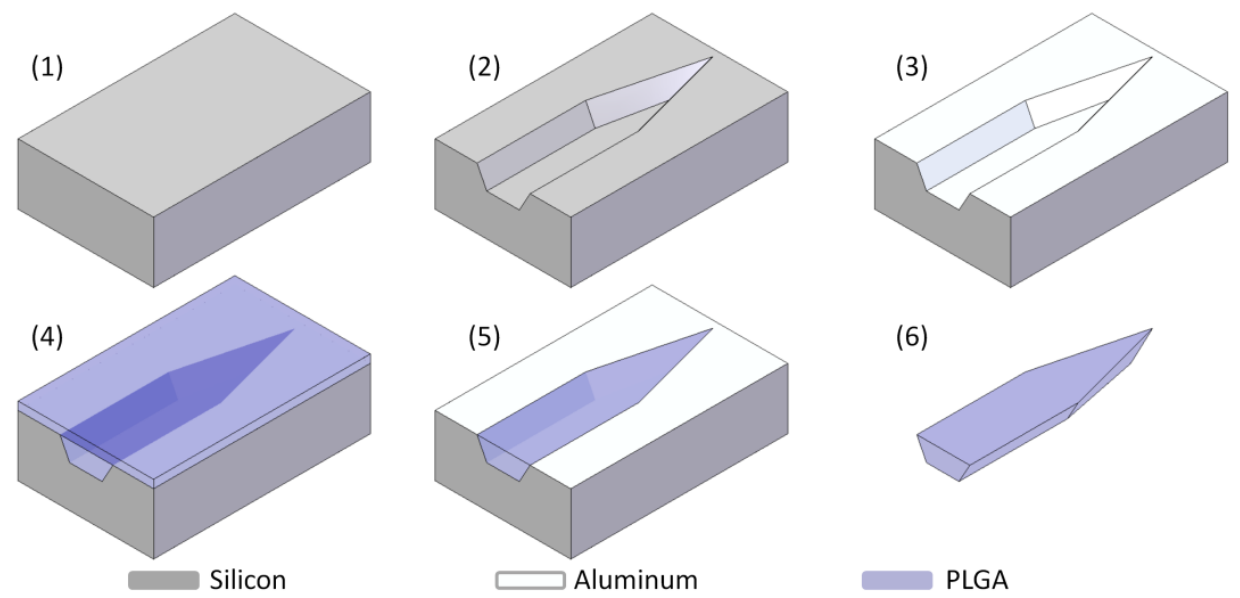

Figure 4: micromolding process. (1) (311) Si wafer. (2) Etching a needle shaped recess. (3) Ti/Al sacrificial layer. (4) PLGA casting and spreading. (5) Polishing (6) Release from mold.

\subsection{Assembly of PLGA needle and non-resorbable backbone}

The needles were put on the wafer that contained the non-resorbable backbone structures and aligned used a pick-and-placer. They were bonded to the wafer simply by heating the assembly to $70^{\circ} \mathrm{C}$ for a few minutes. In case of bending up or down by built-in stress, the needles were first put on an aluminium block at $45^{\circ} \mathrm{C}$, which caused them to relax and to straighten out. The assembly of the PGLA needles and the non-resorbable backbone was then released from the wafer by immersion in $6 \% \mathrm{HCl}$ overnight. Figure 5 shows an assembled device, ready for testing. 


\section{Measurements}

While the molding process had a very high yield, the bonding process was yield-limiting, reducing the total fabrication process yield to about $25 \%$. As a preliminary in vitro test, two of the fabricated needles were inserted in a fresh rat cadaver brain (figure 6). The insertion was done at a speed of 10 $\mu \mathrm{m} / \mathrm{min}$ to a depth of $2 \mathrm{~mm}$, while insertion force was monitored. The needles were $390 \mu \mathrm{m}$ wide at the bottom, and $190 \mu \mathrm{m}$ wide at the top. Two silicon needles of similar size were inserted to serve as a reference. The insertion procedure consisted of four phases: a run-up phase in which the needle does not contact any tissue, followed by an insertion phase in which the force increases linearly with time as the needle enters the tissue. The insertion force needed was about 2 times higher for the PLGA needles than for the silicon needles. After the maximum depth was reached, we waited about two minutes during which the built up tension was seen to relax. During retraction of the needles, a considerable difference between the silicon and the PLGA needles was observed: while retracting silicon needles required less or about equal force than required to insert them, retracting the PLGA required $1.5 x$ to $2 x$ more force. A possible explanation is the fact that films of PLGA tend to take up water and swell with a time constant in the range of a few minutes [27]. 


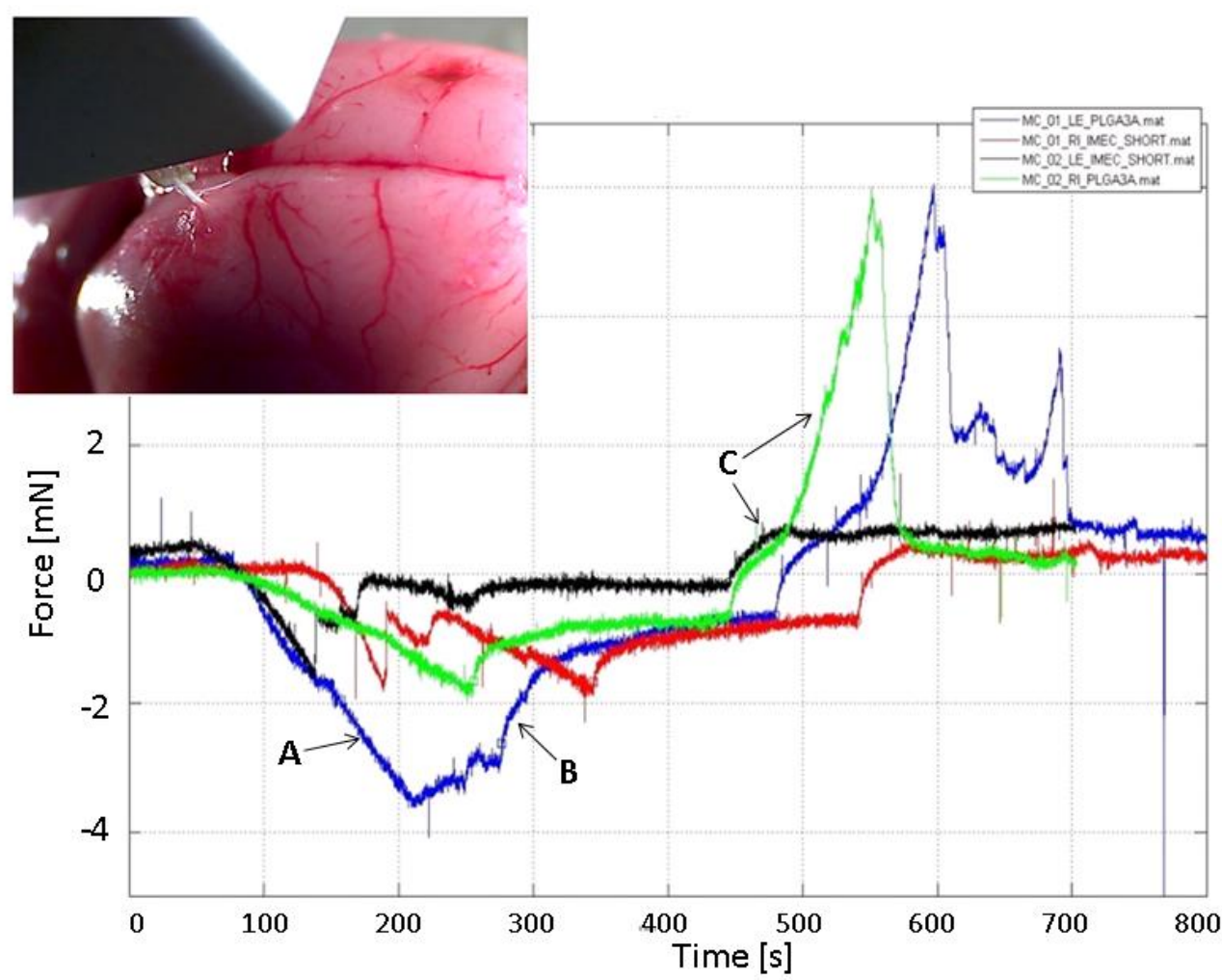

Figure 6: Force measurements during and after insertion of two PLGA microneedles in rat cadaver brain (green, blue), compared with the insertion of two silicon microneedles (red, black). Insertion speed was $10 \mu \mathrm{m} / \mathrm{sec}$. The insertion phase is marked with $A$. In phase $B$, relaxation takes place. During needle retraction (phase C), the PLGA needles seem to stick to the tissue more than the silicon needles.

\section{Discussion and conclusion}

We have shown that (311) silicon is a suitable material to fabricate molds out for the casting of sharp-tipped microneedles. To our knowledge the prior art closest to this is [28], in which razor-blade shaped molds but no pointy needle shapes are fabricated out of (113) Si. No needle shapes were made, though. The molds were utilized to fabricate sharp resorbable microneedles out of low molecular weight PLGA. It was shown to be possible to release the PLGA needles in good shape using an aluminium sacrificial layer and an acidic etching bath. We have also shown that it is possible to bond the fabricated PLGA needles to electrode arrays that are still attached to a wafer, and to release the assembly without adhesion loss.

The assemblies hold promise to be a suitable insertion vehicle for inserting submicrometer-thick electrode arrays in the brain, of which an in vitro insertion test on a cadaver is a first indication. The insertion speed of PLGA microneedles in the brain can be much slower than when using fast dissolving organic materials such as saccharose. The lower dissolution and slower water absorption makes handling in real-life situations more practical. Still, when retracting the needles after 7-8 minutes, already higher forces are needed which indicates interaction with the tissue is already taking place. 
It is likely that other applications may benefit as well from the presented process for molding in (311) Si, an obvious example being drug delivery.

Further fabrication optimisation work should entail the optimisation of the thermal bonding step, which is a bottleneck for the yield of the process. A series of temperatures and pressures could be tested. Larger temperatures and pressures will most probably improve the bonding yield, but could induce more deformation of the PLGA structures. A solution could be to selectively apply pressure to the shafts, but not to the needle tips.

Further characterisation work should also involve electrical characterisation of the electrodes, though standard materials were used that are already well understood. To test the use for the intended application, relatively long term in vivo tests are needed. Only this can lead to a firm conclusion of the suitability of the devices presented here for long term neural recording without substantial scar tissue formation.

\section{Acknowledgments}

This research was made possible by Frederik Ceyssens' postdoctoral research fellowship from FWOFlanders, the KULeuven IDO fund and further supported by the Hercules Foundation for heavy equipment (AKUL 034 and ZW1115). The research leading to these results has received funding from the European Research Council under the European Union's Seventh Framework Programme (FP7/2007-2013) / ERC grant agreement $n^{\circ} 340931$.

\section{References}

[1] BeMent S L, Wise K D, Anderson D J, Najafi K and Drake K L 1986 Solid-state electrodes for multichannel multiplexed intracortical neuronal recording IEEE Trans.Biomed. Eng. 33 23041

[2] Ward M P, Rajdev P, Ellison C and Irazoqui P P 2009 Toward a comparison of microelectrodes for acute and chronic recordings Brain. Res. 1282 183-200

[3] Jorfi M, Skousen J L, Weder C and Capadona J R 2014 Progress towards biocompatible intracortical microelectrodes for neural interfacing applications. Journal of Neural Engineering 12(1) 011001

[4] Polikov V S , Tresco P A and Reichert W M 2005 Response of brain tissue to chronically implanted neural electrodes. Journal of neuroscience methods 148(1) 1-18

[5] Biran R, Martin D C and Tresco P A 2005 Neuronal cell loss accompanies the brain tissue response to chronically implanted silicon microelectrode arrays Experimental neurology 195(1) 115-26

[6] Turner J N, Shain W, Szarowski D H, Andersen M, Martins S, Isaacson M and Craighead H, 1999 Cerebral astrocyte response to micromachined silicon implants Experimental neurology 156, 33-49

[7] Kim Y T, Hitchcock RW, Bridge M J and Tresco P A 2004 Chronic response of adult rat brain tissue to implants anchored to the skull Biomaterials 25(12) 2229-37

[8] Lecomte A, Descamps E and Bergaud C 2018 A review on mechanical considerations for chronically-implanted neural probes Journal of neural engineering 15(3) 031001

[9] Zhou T, Hong G, Fu T M, Yang X, Schuhmann T G, Viveros R D and Lieber C M 2017 Syringe-injectable mesh electronics integrate seamlessly with minimal chronic immune response in the brain. Proceedings of the National Academy of Sciences 114(23) 5894-99

[10] Xiang Z, Yen S C, Xue N, Sun T, Tsang W M, Zhang S, Liao L D, Thakor N V and Lee C 
2014 Ultra-thin flexible polyimide neural probe embedded in a dissolvable maltose-coated microneedle Journal of Micromechanics and Microengineering 24065015

[11] Lecomte A, Castagnola V, Descamps E, Dahan L, Blatché M C, Dinis T M, Leclerc E, Egles C and Bergaud C 2015 Silk and PEG as means to stiffen a parylene probe for insertion in the brain: toward a double time-scale tool for local drug delivery Journal of Micromechanics and Microengineering 25(12) 125003

[12] Kim D H, Viventi J, Amsden J J, Xiao J, Vigeland L, Kim YS, Blanco JA, Panilaitis B, Frechette E S, Contreras D and Kaplan D L 2010 Dissolvable films of silk fibroin for ultrathin conformal bio-integrated electronics Nature materials 9511

[13] Ceyssens F, van Kuyck K, Velde GV, Welkenhuysen M, Stappers L, Nuttin B, Puers R. Resorbable scaffold based chronic neural electrode arrays 2013 Biomedical microdevices. 15(3) 481-93

[14] Ceyssens F, Deprez M, Turner N, Kil D, van Kuyck K, Welkenhuysen M, Nuttin B, Badylak S and Puers R 2017 Extracellular matrix proteins as temporary coating for thin-film neural implants Journal of Neural Engineering 14014001

[15] Armani D K and Liu C 2000 Microfabrication technology for polycaprolactone, a biodegradable polymer Journal of Micromechanics and Microengineering 10(1) 80

[16] Pham P H, Dao D V, Amaya S, Kitada R and Sugiyama S 2007 Fabrication and characterization of smooth $\mathrm{Si}$ mold for hot embossing process IEEJ Transactions on Sensors and Micromachines 127(3) 187-91

[17] Park J H, Allen M G and Prausnitz M R 2005 Biodegradable polymer microneedles: fabrication, mechanics and transdermal drug delivery Journal of Controlled Release 104(1) 51-66

[18] Luttge R, Berenschot E J, De Boer, M J, Altpeter D M, Vrouwe E X, Van Den Berg A, and Elwenspoek M, 2007 Integrated lithographic molding for microneedle-based devices. Journal of microelectromechanical systems 16(4) 872-84

[19] Andersen T E, Andersen A J, Petersen R S, Nielsen L H and Keller S S 2018 Drug loaded biodegradable polymer microneedles fabricated by hot embossing Microelectronic Engineering 195 57-61

[20] Narayanan S P and Raghavan S 2017 Solid silicon microneedles for drug delivery applications The International Journal of Advanced Manufacturing Technology 93 407-22

[21] Pal P, Sato K, Gosalvez M A, Tang B, Hida H and Shikida M 2010 Fabrication of novel microstructures based on orientation-dependent adsorption of surfactant molecules in a TMAH solution Journal of Micromechanics and Microengineering 21(1) 015008

[22] Ceyssens F and Puers R 2015 Insulation lifetime improvement of polyimide thin film neural implants Journal of neural engineering 12054001

[23] Zubel I and Kramkowska M 2004 Possibilities of extension of 3D shapes by bulk micromachining of different $\mathrm{Si}$ (hkl) substrates. Journal of Micromechanics and Microengineering 15(3) 485

[24] Moldovan N and Divan R 2014 Si (100) and Si (311)-A comparative study for nanofabrication. In Nanotechnology (IEEE-NANO), 2014 IEEE 14th International Conference on. IEEE. 212-20

[25] Lin C C, Yang C C, Ger J, Deng J F and Hung D Z 2010 Tetramethylammonium hydroxide poisoning Clinical toxicology 48(3) 213-7

[26] Biswas K and Kal S 2006 Etch characteristics of KOH, TMAH and dual doped TMAH for bulk micromachining of silicon Microelectronics journal 37(6) 519-25

[27] Sharp J S, Forrest J A and Jones R A L 2001 Swelling of poly (DL-lactide) and polylactide-coglycolide in humid environments Macromolecules 34(25) 8752-60

[28] Werkmeister J, Gosalvez M A, Willoughby P, Slocum A H and Sato K 2006 Anisotropic etching of silicon as a tool for creating injection molding tooling surfaces Journal of microelectromechanical systems 15(6) 1671-80 